\title{
Untersuchung über die periphere Bahn des N. vagus.
}

I. Mitteilung. Die markhaltigen Fasern des rechten Vagus.

Von

\section{Dr. Yoshio Iwama.}

Aus dem Anatomischen Institut der Kaiserlichen Universität zu Kyoto

(Leiter: Professor S. Funaoka).

Mit 8 Figuren auf Tafeln XVII-XVIII und 1 Textfigur.

\section{Inhaltsverzeichnis.}

I. Einleitung.

II. Untersuchungsmaterial und Untersuchungsmethode.

III. Anastomose zwischen N. vagus und N. sympathicus am Halsteile.

IV. Durchschneidungsversuch des N. vagus am Halsteile.

1. Die Degeneration im Vagusstamme.

2. Die Dégeneration in der Anastomose.

3. Die Degeneration im N. sympathicus am Anastomosenteile.

4. Die Degeneration des Sympathicusstammes am Halsteile.

5. Die Degeneration im Truncus sympathicus abdominalis.

6. Die Degeneration in den Nn. splanchinici.

V. Durchschneidungsversuch des Sympathicusstammes am Halsteile.

VI. Zusammenfassung.

Literatur.

Tafel und Tafelerklärung.

\section{Einleitung.}

Der periphere Verlauf der Vagusfasern, die verschiedene Bauch- und Beckenorgane innervieren, ist durch physiologische und pharmakologische Untersuchungen erschlossen worden, aber auf anatomischem Wege lässt er sich nur bis zum Magen oder bis zum Ganglion semilunare verfolgen.

In der Brusthöhle ist der Vagus mit dem Sympathicus in vielfachen Verbindungen. In der Bauchhöhle bilden die beiden Nerven ein so dichtes Geflecht, dass man sie kaum von einander trennen kann. Diese verwickelten Verbindungen sind für die beiden Nerven genenseitig, der Vagus gibt einerseits an den Sympathicus seine Fasern ab und anderer- 
seits erhält er von dem letzteren sympathische Fasern. Edgeworth hat die Fasern von 1.8-6.3 $\mu$ im Truncus sympathicus unter dem Namen „Vagusfasern“ zusammengebracht. Aus dieser Benennung scheint es, dass dieser Autor eine gewisse Beziehung dieser Fasern zum Vagus sich vorgestellt hat. Bei gewissen Tieren wie beim Hunde sind die beiden Nerven am Hals von einer und derselben Epineuralscheide umgeben und gehen erst dicht oberhalb der Brustapertur von einander ab. Der Vagus und der Sympathicus eines solchen Tieres sind nicht für gleichwertig gegen die Nerven der anderen zu halten, bei denen auch am Hals die beiden Nerven getrennt sind. Man fasst richtiger bei den ersten Sorten von Tieren den Vagus und den Sympathicus als die Endzweige vom N. vagosympathicus auf, während bei den anderen Tieren der Vagus und der Sympathicus für sich selbst einen isolierten Nerven darstellen. Die Katze gehört zu der zweiten Gruppe, und die beiden Nerven dieses Tieres ziehen vom Hals als zwei Nerven ab, trotzdem gewisse Anastomosen zwischen ihnen fast immer nachzuweisen sind. Die Bedeutung dieser Anastomosen wird von mir jetzt in Frage gestellt.

\section{Untersuchungsmaterial und Untersuchungsmethode,}

Als Versuchtier habe ich die Katze aus dem Grunde vorgezogen, dass bei diesem Tier der N. vagus einerseits am Hals ziemlich dick und vom Sympathicus leicht zu trennen ist, und andererseits die Katze den operativen Eingriffen sehr gut widersteht.

Um den peripheren Verlauf der markhaltigen Fasern zu bestimmen, habe ich den Vagus am Hals durchgeschnitten und mittels Marchi'scher Methode die dadurch hervorgerufenen Degenerationsbilder, die sog. „W Waller'sche Symptome " peripherenwärts verfolgt.

Die Gesamtzahl der Versuchstiere ist 8, von denen ich 2 für die Kontrollierungs- und 6 für die eigentlichen Durchschneidungsversuche benutzte. Das 'Tier wurde schwach chloroformiert, und nach der Desinfektion hat man den rechten Vagusstanm am unteren Cervicopharingealteil durchgeschnitten. Nach 7-10 Tagen wurde das Tier abgetötet und unter sorgfältiger Vermeidung von Schütteln, Ziehen oder Quetsche des Nerven diesen von den anderen Körperteilen befreit und in Müllersches Gemisch hineingelegt. Nach mehr als einer Woche wurde der Nerv unter mehrmaliger Wechselung der Fixierflüssigkeit herauspräpariert und 3-6 Tage lang in die Marchische Lösung gehalten, danach 24 Stunden lang mit fliessendem Wasser gewaschen, entwässert, im Paraffin eingebettet, and nun wurden Langs- und Querschnittserien von 
15-20 $\mu$ Dicke hergestellt. Hierbei habe ich den Gebrauch von Xylol und Xylol-haltigen Substanzen für alle die obenerwähnten Prozesse absolut vermieden, damit ich die Artefacte und die daraus resultierenden Fehlerquellen des mikroskopischen Bildes möglichst ausschliessen könnte. Zur Paraffindurchtränkung habe ich also Chloroform und zur Entparaffinierung Petroleumbenzin vorgezogen. Um die Befunde in den Schnittpräparaten zu versichern, habe ich die mit Marchischer Lösung vorbehandelten Nervenfasern in Glyzerin zerzupft und ich bin nun von der Uebereinstimmung der Resultate der beiden Methoden überzeugt.

\section{Die Anastomose zwischen N. vagus und N. sympathicus am Halsteile.}

Die Angaben von Autoren über die Anastomose zwischen Vagus und Sympathicus bei der Katze sind abweichend. Boehm gibt an, dass die Nervi vagi und sympathici bei der Katze vom Ganglion cervicale supremum ab bis zum Ganglion cervicale medium dicht nebeneinander verlaufen und von einer gemeinsamen Bindegewebshülle umschlossen sind, während aber die künstliche Trennung dieser beiden Nerven an der Katze sehr leicht ist und ohne erhebliche mechanische Insultierung der Nerven mit einem stumpfen Haken ausgeführt werden kann. Weiter sind nach ihm Verbindungen zwischen beiden Nerven in der Gegend des Ganglion cervicale medium regelmässig nachweisbar. Fischer fand auch keine Anastomose zwischen den Nerven, sie sind nur locker zusammengehalten. Nach Sternschein finden sich aber bei histologischer Untersuchung zahlreiche markhaltige Anastomosen auch in jenen Fällen, wo makroskopisch zwischen den isoliert gelegenen Ganglien nur Bindegewebe erkennen lassen, und auch im weiteren Verlauf der beiden Nerven gegen die obere Brustapertur konnten stets Verbindungen gefunden werden, deren Stärke, Zahl und Lage aber sehr variabel erscheinen. Mit grosser Regelmässigkeit jedoch finden sich zwei Anastomosen, von denen die eine knapp unterhalb des Eintrittes des Nerven ins Ganglion, die zweite etwas unterhalb des Ganglion cervicale inferius lag.

Die Untersuchungen von S. Biffi, Cl. Bernard und J. Budge über den Einfluss durch Reizung des Sympathicus am Halsteile auf die Gefässerweiterung widersprechen den von W. Beid, Onuf und Colling mit dem gleichen Zwecke gemachten, aber diese Tatsache ist schon hinreichend, um den Verdacht zu erregen, dass vielleicht wenigstens ein Teil von gefässerweiterunden Nervenfasern nach ihrem Austreten aus der autonomen Leitung in der Medulla oblongata durch irgendwo vorhandene Anastomose in den Halssympathicus übertrete. 
Die Untersuchungen über diese Frage von Carson, Boem, Roeber, Reighard und Jennings u. a. bestätigten drei Anastomosen zwischen dem Vagus und dem Sympathicus: namentlich die erste Anastomose zwischen dem Ganglion cervicale superius und dem Ganglion nodosum, sodann die zweite zwischen den beiden Nervenstämmen etwas unterhalb der ersten Anastomose und schlieslich die dritte zwischen dem Sympathicusteil etwas oberhalb des Ganglion cervicale inferius und dem Vagusstamme.

Durch Bestätigung dieser Anastomosen ist die Gefässkontraktion nach der Reizung des Halssympathicus klar geworden, aber über das Wesen der manchmal durch die Reizung des Halssympathicus hervorgerufenen Gefässerweiterung an der Kopfhalsgegend weiss man nichts Sicheres.

Um diese Frage zu lösen, muss man zunächst entscheiden, ob der Vagus wirklich die dilatatorischen Nerven den betreffenden Giegenden liefert, oder ob die oben erwähnten verschiedenen Erscheinungen den Funktionen der eigentlichen sympathischen Nervenfasern des Sympathicus oder denen der durch die Anastomose dahin eintretenden Fasern des Vagus zuzuschreiben seien.

An der dritten Anastomose have ich bei der Katze folgende Resultate bekommen. Auf der rechten Seite konnte ich an allen acht Tieren die Existenz von Anastomosen klar nachweisen, habe aber im Widerspruch mit der Angabe von Sternschein die Anastomose immer zwischen dem Vagusstamme und den sympathischen Ganglien gefunden.

Auf der linken Seite konnte ich nur bei 4 Tieren unter 8 die Anastomose sicher nachweisen. Unter 8 Tieren war ber 2 gar kein Anastomose vorhanden und bei anderen 2 war es zweifelhaft.

Die Stärke der Anastomose war auf der linken Seite bei weitem schwächer als auf der rechten. Näheres über diese Anastomosen möchte ich an anderer Stelle mitteilen.

Die anatomische und physiologische Bedeutung dieser Anastomose ist noch nicht klar. Diese Arbeit beabsichtigt, diese Verbindung und den weiteren Verlauf der Vagusfasern klarzulegen.

\section{Durchschneidungsversuch des N. vagus am Halsteile.}

\section{Die Degeneration im Vagusstamme.}

Ueber das Wesen der durch die Nervendurchschneidung bedingten, sogennanten Wallerschen Degeneration der peripheren Nervenfasern und besonders der markhaltigen gibt es sehr viele Meinungen, die zwar von einander verschieden, aber darin übereinstimmend sind, dass für die 
Diagnose dieser Degeneration die Existenz der durch das „Marchische Verfahren" geschwärzten Osmiumgranula notwendig und hinreichend ist.

Diese Tatsache habe ich daher als das Diagnosenmerkmal benutzt.

O. Larsell und M. L. Mason zogen aus ihrer Untersuchung über die durch die Durchschneidung des Vagus am mittleren Halsteil bedingte Degeneration den Schluss, dass die Degeneration der Nervenfasern durch die Durchschneidung von zentrifugaler, aber nicht von zentripetaler Natur sei.

Am Hals besteht der Vagus aus drei Arten von Nervenfasern, d. h. aus den vielen markhaltigen, von denen die einen breite und die anderen schmale Markscheiden haben, und aus den wenigen marklosen. Verfolgt man nun den Vagus weiter abwärts, so sieht man, dass die dicken Markfasern sich hauptsächlich zu den Halsorganen wenden. Die Fasern, welche zum Herzen ziehen, enthalten neben vereinzelten dicken zahlreiche zarte Markscheiden und schon sehr viele nackte Achsencylinder. Weiter abwärts nehmen die dicken Fasern immer mehr an Zahl ab, und an der Cardia besteht der Vagus grösstenteils aus feinen Markfasern und nackten Achsenzylinderm.

Die Resultate der Durchschneidungsversuche sind bei allen sechs Katzen übereinstimmend, von denen drei sieben Tage nach der Durchschneidung, und die anderen drei nach zehu Tagen getötet sind. Ich werde daher im Nachstehenden an einer Katze No. 5, die sieben Tage nach der Operation getötet wurde, das Degenerationsbild schildern.

Gleich unterhalb der Durchschnittsstelle (Fig. 1) fallen fast alle die markhaltigen Nervenfasern im Vagusstamme der Degeneration anheim, namentlich sieht man auf dem Längsschnittspräparate dem Verlauf der Nervenfasern entlang eine grosse Menge Osmiumkörner von verschiedener Grösse und Form in Reihen abgelagert, und man kaun fast keine von Degenerationsschollen freien markhaltigen Nervenfasern finden. Diese Osmiumkörner kann man aber von den durch Osmium verschwär"zten Fettkügelchen, die zufällig zwischen Nervenfasern verlagert sind, an dem verschiedenen Aussehen leicht unterscheiden. An den degenerierten Nervenfaasern sieht man noch, dass die Myelinscheiden stark zerstört sind und unregelmässige Figur tragen.

Die Veränderung des Nervenfasern des Vagusstammes unterhalb der oben erwähnten Anastomosen ist dicht unterhalb des Durchschneidungsortes fast gleich, und man sieht da auch viele Osmiumkörnchen.

An der Cardia des Magens (Fig. 2) ist der rechten Vagus (Chorda posterior vagi) an markhaltigen Nervenfasern beträchtlich ärmer als der obere 'Teil, und folglich sind die Osmiumkörnchen nicht zahlreich zu finden, diese liegen da nur in wenigen Reihen. 
Der rechte Vagus zieht nach dem Durchgang durch das Zwerchfell, zur grossen Kurvatur des Magens zahlreiche Nervenfasern abgebend, nach Ganglion solare hin.

Gleich oberhalb des Ganglion solare sind nur wenige Marchische Schollen zu sehen, man weist nur mit grosser Mühe die Existenz von degenerierten Fasern nach.

\section{Die Degeneration in der Anastomose.}

Das Degenerationsbild der Nervenfasern in der Anastomose ist durch die Vielgestaltigkeit der letzteren nicht einfach, aber bei allen sechs operierten Tieren konnte ich in irgendwelchen Anastomosenästen zwischen dem Halsvagus und dem Sympathicus auf der rechten Seite degenerierte Fasern finden. Ich möchte als Beispiel im Nachstehenden die Degeneration in den Anastomosenästen bei der Katze No. 5 näher beschreiben.

An der untersten (dritten) Anastomose zwischen dem Vagus und dem

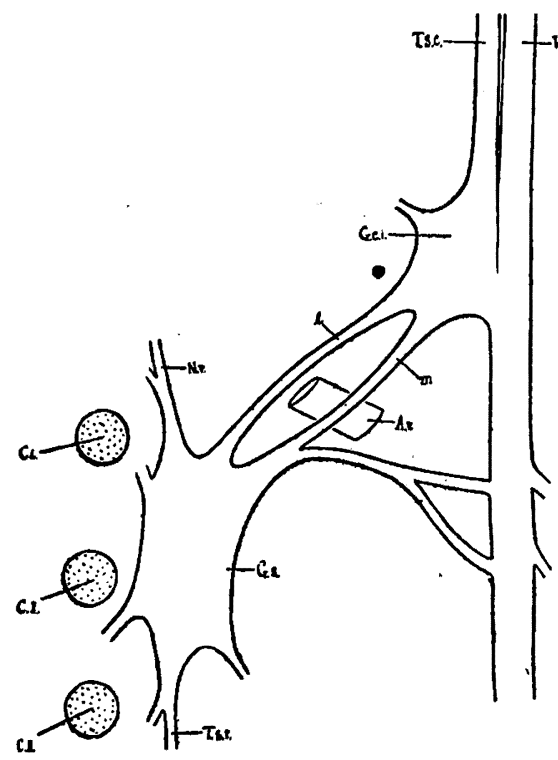

Die schematische Darstellung der unteren Halsanastomose zwischen $N$. vagus $u$. N. sympathicus auf der rechten Seite bei der Katze No. 5.

V. Vagus, T.s.c. Truncus sympathicus cervicalis, G. c. i., Ganglion cervicale inferius, 1, lateraler Nerv, m., medialer Nerv, A. v. Arteria vertebralis, N. v. N. vertebralis, G.s., Ganglion stellatum, T. s.t. Truncus sympathicus thoracicus, $\mathrm{Cl}$. Clavicula, C.I. Costa I, C. II. Costa II. Sympathicus bei der Katze No. 5 sieht man keinen nennenswerten Ramus anastomoticus, der Vagusstamm liegt dicht am Ganglion cervicale inferius an, und die beiden Nerven sind dabei teilweise verwachsen (Fig. 3). Vom Ganglion cervicale inferius dextra gehen zwei Nerven hervor und ziehen zum Ganglion stellatum hin. Der mediale Nerv nimmt in sich auf seinem Wege zum Ganglion stellatum einen Anastomosenast auf, der mit zwei Wurzeln von dem Vagus entspringt.

Von diesen beiden Nerven konnte ich im lateralen Nerven auf dem Längsschnittpräparate seinem Verlauf entlang ziemlich zahlreiche Osmiumkörnchen finden : auch im medialen konnte ich ebenfalls die Existenz von diesen nachweisen, aber die Menge im letzteren ist etwas geringer als im vorigen. Dagegen konnte ich in den Anastomosen zwi- 
schen dem Vagusstamm und dem medialen Nerv gar keine Osmiumkörner nachweisen.

Nach den obigen Befunden verlaufen die markhaltigen Vagusfasern durch die Anastomose zwischen dem Stamme und Ganglion cervicale inferius in den Truncus sympathicus hinein.

\section{Die Degeneration im N. sympathicus am Anastomosenteile.}

Ober- und unterhalb der untersten (dritten) Halsanastomose konnte ich im Truncus sympathicus degenerierte Nervenfasern nachweisen, deren Menge oberhalb der Anastomose viel geringer als unterhalb derselben ist.

Im Halssympathicus oberhalb der Anastomose (Fig. 4) konnte ich auf Längsschnitten dem Verlauf der Nervenfasern entlang eine ziemlich ansehnliche Menge von etwas kleineren Osmiumkörnchen beobachten. Weil diese Körnchen wie diejenigen im Vagus gleich beschaffen sind, kann ich daraus schliessen, dass der Halssympathicusstamm auch oberhalb dieser Anastomose degenerierte Nervenfasern enthält. Weil ich bei der Durchschnieidung des Vagus am Halsteile mit aller Sorgfalt die Verletzung des Sympathicus vermied, ist diese Degeneration der Nervenfasern nicht durch einen Fehler der Operatton entstanden, sondern sicher durch die Existenz der vom Vagus stammenden Nervenfasern hervorgerufen.

Auch konnte ich auf den Längsschnitten des N. vertebralis ausser vielen nicht degenerierten markhaltigen Nervenfasern dem Verlauf der Nervenfasern entlang eine relativ grosse Menge von verhältnismässig grossen Osmiumkörnern beobachten.

Diese Tatsache weist darauf hin, dass auch hier markhaltige Nervenfasern vom Vagus vorhanden sind.

\section{Die Degeneration des Sympathicusstammes am Halsteile.}

Im rechten Ganglion stellatum wird deutlich die Wallersche Degeneration nachgewiesen, auch sieht man im Brustsympathicus viele mit Osmiumkörnern beladene Nervenfasern.

Am rechten Brustsympathicus konnte ich über seine ganze Länge hin deutlich die Existenz der degenerierten Nervenfasern nachweisen, z. B. beobachtete ich in der Höhe vom sechsten Intercostalraum (Fig. 5) ohne Zweifel im Brustsympathicus zahlreiche dicke degenerierte markhaltige Nervenfasern ; noch weiter abwärts scheinen die Körner an Zahl abzunehmen, ich fand in der Höhe des elften Intercostalraums die Osmiumkörner etwas geringer an Zahl und etwas kleiner an Gestalt. 
Nebenbei konnte ich auch am Brustteile des linken Truncus sympathicus oberhalb der Verzweigungsstelle der linken Eingeweidenerven eine kleine Anzahl von degenerierten markhaltigen Nervenfasern beobachten, aber die Frage, ob diese markhaltigen Nervenfasern vom rechten Vagus durch das Ganglion solaris oder irgendandere in der Brust zwischen Vagus und Sympathicus vorhandene direkte oder indirekte Anastomosen in den linken Brustsympathicus eingetreten sind, wird durch eine zukünftige Untersuchung entschieden werden.

\section{Die Degeneration im Truncus sympathicus abdominalis.}

Am oberen Teil des rechten Bauchsympathicusstammes (Fig. 6) konnte ich die Existenz von degenerierten markhaltigen Nervenfasern bestätigen; da sind aber die Osmiumkörner kleiner, geringer an Zahl und hier und da nur zerstreut vorhanden; man sieht daher, dass die Vagusfasern im Sympathicus vor dem Durchtritt durch das Zwerchfell grösstenteils den Truncus sympathicus zum Ganglion solare verlassen.

Am Stamm des linken Brustsympathicus konnte ich auch wenige degenerierte markhaltige Nervenfasern beobachten, aber über die Verlaufsbahn dieser Fasern bin ich nicht im Stande, etwas Festes zu sagen.

\section{Die Degeneration in den Nn. splanchinici.}

Im rechten grossen und kleinen (Fig. 7) Eingeweidenerven konnte ich über die ganze Länge hindurch degenerierte markhaltige Nervenfasern beobachten. An diesem Teile sind die Osmiumkörner verhältnismässig gross und zahlreich und liegen dicht nebeneinauder, sie sind aber im kleinen Eingeweidenerven geringer als im grossen.

Im linken grossen (Fig. 8) und kleinen Eingeweidenerven konnte ich auch relativ zahlreiche dicke degenerierte markhaltige Nervenfasern finden.

\section{Der Durchschneidungsversuch des Sympathicusstammes am Halsteile.}

Auf Grunde der oben erwähnten Betrachtungen scheinen im Truncus sympathicus der Katze die markhaltigen Nervenfasern vom Vagus beimischend vorhanden zu sein. Um das festzustellen muss man sich davon überzeugen, dass die Degeneration im Sympathicus nach der Durchschneidung des Vagus nicht davon herrürt, dass die im Sympathicus enthaltenen markhaltigen Fasern von anderer Herkunft bei der Operation mitgeschädigt sind und secundäre Degeneration ihrer Markscheiden verursacht haben. 
Zu diesem Zwecke have ich bei einer Katze anstatt des Vagus den Truncus sympathicus am Hals durchgeschnitten und überraschende Befunde sowohl am Sympathicus als auch am Vagus getroffen.

Nach zehn Tagen der Durchschneidung des rechten Sympathicusstammes oberhalb der untersten (dritten) Halsanastomose zwischen Vagus und Sympathicus brachte ich die Versuchskatze durch die Narkose zum Tode und nach verschiedenen obigen Behandlungen gelangte ich zu der Mikroskopierung. Im Truncus sympathicus war unterhalb der Durchschneidungsstelle (Fig. 8) eine kleine Anzahl degenerierter markhaltiger Nervenfasern vorhanden, die noch weiter abwärts unterhalb des gleichseitigen Gangliou stellatum nachzuweisen sind.

Im rechten Vagusstamme konnte ich ober- und besonders unterhalb dér betreffenden Anastomose eine grosse Menge Osmiumschollen dicht nebeneinander liegen sehen, aber je weiter nach unten, desto geringer wurde die Menge, bis ich schlieslich am unteren Brustvagus überhaupt keine mehr nachweisen konnte.

Im gleichseitigen $\mathrm{N}$. vertebralis konnte ich relativ zahlreiche Osmiumkörner beobachten, während ich sie in den Eingeweidenerven der rechten Seite nur wenig nachweisen konnte.

Die Untersuchung der Degeneration im Sympathicus und Vagus nach der Durchschneidung des Halssympathicus habe ich bis jetzt nur bei einer Katze ausgeführt und werde Weiteres an anderer Stelle angeben.

Nach dem obigen Versuche sind.die degenerierten Fasern im Truncus sympathicus nach der Durchschneidung des Halssympathicus viel weniger zahlreich als nach der Vagotomie, und dies bedeutet, dass viele Vagusfasern sicher im Brustsympathicus enthalten sind. Ausserdem sieht man, dass die im Halssympathicus enthaltenen markhaltigen Nervenfasern teilweise durch die dritte Anastomose in den Brustvagus übergehen. Die Herkunft dieser Fasern ist noch nicht festgestellt.

Herr Prof. S. Funaoka besass die grosse Güte, mich bei dieser Arbeit freundlich zu beraten, wofür ich an dieser Stelle einen ergebensten Dank aussprechen möchte.

\section{Zusammenfassung.}

Die Resultate der Durchschneidungsversuche des rechten Vagus der Katze sind wie folgt.

1) Ein Teil der markhaltigen Nervenfasern des rechten Vagus geht durch den gleichseitigen Vagus in das Ganglion solare des Bauchsympathicus. 
Ein anderer Teil geht durch die unterste (dritte) Halsanastomose zwischen Vagus und Sympathicus in den Stamm des Halssympathicus über. Diese Fasern steigen im Truncus sympathicus teils auf und teils ab.

2) Ein Teil von den absteigenden markhaltigen Nervenfasern aus dem Vagus tritt durch das gleichseitige Ganglion stellatum hindurch in den gleichseitigen Plexus brachialis ein.

3) Der andere Teil zieht den rechten Brustsympathicus entlang herunter und tritt durch die gleichseitigen Eingeweidenerven in das gleichseitige Ganglion solare des Bauchsympathicus ein.

4) Ein Teil von den markhaltigen Nervenfasern, die im rechten Brustsympathicus nach abwärts gelaufen sind, tritt in den rechtsseitigen Bauchsympathicusstamm ein und zieht darin noch weiter herab.

5) Die linken Eingeweidenerven enthalten auch die markhaltigen Nervenfasern des rechten Vagus.

6) Auch der Stamm des linken Brust- und Bauchsynupathicus enthält einen Teil der markhaltigen Nervenfasern des rechten Vagus. Aber über den Verlauf dieser Nervenfasern ist noch nichts entschieden worden.

7) Ein Teil der markhaltigen Nervenfasern des rechten Halssympathicusstammes tritt durch die unterste (dritte) Halsanastomose zwischen Vagus und Sympathicus in den gleichseitigen Vagusstamm ein, und danach steigt der grösste Teil dieser Fasern auf und der übriggebliebene ab.

\section{Literatur.}

1. Bay on, P. G., Die histologischen Untersuchungsmethoden des Nervensystems.

2. Boehm, R., Arch. f. exper. Pathol. u. Parmakol. Bd. 4, S. 225, 351. 1875.

3. Bersou. W., Phénomènes de dégénérescence et de régénérescence consécutifs à la section et à la ligature d'un nerf périphérique. Nérvaxe, Vol. 14, 15, p. 339-423.

4. Biondi, G., La degenerazione walleriana dei nervi periferici, particolarmente studiata dal lato istochimico ed il valore degli attuali metodi dindagine per la dimostrazione istochimica di sostanze grasse e lipoid. Fol. neurobiol., Bd. 7, 1913. (Cie. in Jahresber. d. Anat. u. Entwicklungsgesch. Bd. 19A, S. 287. 1913.)

5. Bernard, Cl., Cpt. rend. des śeances de la soc. de biol. S. 163. 1851. (nach E. Sternscheins, , Ueber Anastomose zwischen Vagus und Sympathicus der Katze". Zeitschr. f. Anat. u. Entwicklungsgesch. Bd. 64. 1922.) 
6. Carson, A. J., Americ. Journ. of Physiol. Vol. 30, p. 408. 1912.

7. Cajal, S., Ramón y, Estudios sobre la degeneración y regeneración del sistema nervioso. (cir in Jahresber. d. Anat. u. Entwicklungsgesch. Bd. 20A, S. 203. 1914.)

8. Chase, M. R. and Ranson, S. W., The structure of the roots, trunk and branches of the vagus nerve. Journ. of compar. neurol. Vol. 24, 1914.

9. Clerk, E., Regeneration of medullated nerves in the absence of embrionic nerve fibres, following experimental nontraumatic degeneration. Journ. of compar. ne'urol. Vol. 24, 1914.

10. Donaggi, O. A., Die beginnende Degeneration der Nervenfasern. Jahresber. der Anat. u. Entwicklungsgesch. Bd. 19A, S. 283. 1913.

11. Edgeworth, M. B., On a Large-fibred Sensory Supply of the Thoracic and Abdominal Viscera. Journ. of Physiol. Vol. 13, S. 260-271. 1892.

12. Fischer, J., Vergleichende anatomischẻ und histologische Untersuchung über den Sympathicus usw. Diss. Zürich, 1904.

13. Gaskell, W. H., On the structure, distribution and function of the nerves which innervate the visceral and vascular systems. Journ. of physiol. London, Vol. 7, p. 19. 1886.

14. Hoffmann u. Sahwalbe, Anatomie des Menschen. Bd. II, S. 317. Methoden' der Neurologischen Forschung.

15. Jaçobsonn, L., Anatomische Veränderungen des Nervensystems nicht pathologischer Natur. Handbuch der pathologischen Anatomie des Zentralnervensystems. Berlin, Bd. 1, S. 96. 1904.

16. Kohn, A., Ueber die Entwicklung des sympathischen Nervensystems der - Säugetiere. Arch. f. mikr. Anat. Bd. 70. 1907.

17. Copper, A., Vergleichende Anatomie des Nervensystems.

18. Loewenthal, N., Contribution expérimentale à l'étude de la dógénérescence rétrograde. Nérvaxe, Vol. 14, 15. 1913. (Aus Jahresber. d. Anat. u. Entwicklungsgesch. Bd. 19, S. 283)

19. Larsell, O., Nerve termination in the lung of the rabbit. Journ. of compar. neurol. Vol. 33, S. 105-129. 1921.

20. Mott, The Croonian Lectures on the Degeneration of the Neurone. The British medical Journal 1900, Bd. 1, S. 15-17. (Aus Bayon, Die histologischen Untersuchungs Methoden des Nervensyste s.)

21. Michailow, S., Versuch einer systematischen Untersuchung der Leitungsbahn des sympathischen. Nervensystems. Experimentelle, anatomischpathologische Untersuchung des Gebietes des Ganglion stellatum und Ganglion cervicale inferius. Pflügers Archiv. Bd. 128. S. 283-397. 1909.

22. Molhault, M., La nerf vague. Le Nervaxe, Vol. 11, p. 137. 1910.

23. Möllgaard, H., Studien über das respiratorische Nervensystem bei den Wirbeltieren. Skandin. Arch. f. Physiol. Bd. 26, S. 315-385. 1912.

24. Mohlhaut, M., Les ganglions périphériques du vague. Le Nérvraxe, T. 1., p. 525-579. 1913.

25. Mohlhaut, M., Le nerf vague, Etude anatomique et expérimentale. Louvain. 1914. 
26. Müller, L. R., Die Lebensnerven. 1924. Histologie des sympathischen Grenzstranges und seiner Ganglionknoten.

27. Müller, L. R., Die Lebensnerven. 1924. Beiträge zur Anatomie, Histologie und Physiologie des $\mathbf{N}$. vagus.

28. Nisse., Histotogie und histopathologische Arbeiten. Bd. IV, S. 484. Die wallersche Degeneration.

29. Onodi, A. D., Ueber das Verhältniss des cerebrospinalen Faserbündels zum Eympathischen Grenzstrang. Arch. f. Anat. u. Physiol. 1884.

30. Onuf u. Collins, Arch. of neurol. a. psychopath. Bd. 3, p. I. 1900. (Aus dem Aufsatz E. Sternschein s: ,, Ueber Anastomose zwischen Vagus und Sympathicus der Katze “. Zeitschr. f. Anat. u. Entwicklungsgesch. Bd. 64, 1922.)

31. Roeber, Untersuchung des Nerveneinflusses auf die Blutgefässe. Rostock 1869.

32. Reid, W., Journ. of physiol. Bd. 17, p. 483.1895.

88. Reighard, J. u. Jenning8, H. S., Anatomie of the cat. New York 1903.

34. Raimann, Zur Technik der Marchi-Methode. Neurologisches Zentralblatt. Bd. 20, S. 608-609. 1901.

35. Ranson, S. Walter, The structure of the Vagus Nerve of man as demonstrated by a Differential Axon Stain. Anat. Anz. Bd. 46. p. 522-525. 1914.

36. Serafino Biffi, Intorno all'influenza che hanno sull'occhio $i$ due nervi: Grande simpathico e Vago. Diss. Paris 1848.

37. Schumacher, S. V., Die Herznerven der Säugetier und des Menschen. Sitzungsber. d. Akad. Wien, Mathem.-naturw. KI. III, Abs. III, I. 1902.

38. Stranskys, Zur Konservierung von Faserfärbung. Neurol. Zentralblatt 1901, Bd. 20, S. 983.

84. Spielmeyer, W., Die Fehlerquellen der Marchischen Methode. Zentralblatt für Nervenheilkunde 1903, Bd. 26, s. 457.

35. Stranskys, Bemerkung über die bei Marchi-Färbung auftretenden artifiziellen Schwärzungen. Neurol. Zentralblatt 1903, Bd. 22, S. 658.

36. Sternschein, E., Ueber Anastomase zwischen Vagus und Sympathicus der Katze. Zeitschr. f. Anat. u. Entwicklungsgesch. Bd. 64, S. 441. 1922.

\section{Tafelerklärung (XVII-XVIII).}

Fig. 1. Katze No. 5. Längsechnitt durch den rechten $\mathbf{N}$. vagus dicht unterhalb der Durchschnittsstelle, nach Marchischer Methode. Leitz Obj 3. Ocul. I. T. L. $160 \mathrm{~mm}$.

Fig. 2. Katze No. 5. Längsschnitt duroh den rechten $\mathbf{N}$. vagus an Cardia des Magens, nach Marchischer Methode. Leitz Obj. 7. Ocul. I. T. L. 160mm. 
Fig. 3. Katze No. 5. Die untere Halsanastomose zwischen dem rechten N. vagus uncl dem Ganglion cervicale inferius, nach Marchischer Methode. Leitz Obj. 3. Ocul. I. T. L. $160 \mathrm{~mm}$.

a. Ganglion cervicale inferius.

b. Die Anastomose.

c. N. vagus.

Fig. 4. Katze No. 5. Längsschnitt durch den rechten Halssympathicus oberbalb dיI Inastomose, nach M a reh ischer Methode. Leitz Obj. 7. Ocul. I. T. L. $160 \mathrm{~mm}$.

Fig. 5. Katze No. 5. Lüngsschnitt durch den rechten Brustsympathicus in der Höhe vom sechsten Intercostalraum, nach Marchischer Methode. Ieitz Obj. 7. Ocul. I. T. L. 160mm.

Fig. 6. Katze No. 5. Längsschnitt durch den oberen Teil des rechten Bauchsympa- thicus, nach Marchischer Methode. Leitz Obj. 7. Ocul. I. T. L. 160mm.

Fig. 7. Katze No. 5. Längsschnitt durch den rechten kleinen Eingeweidenerven (Splanchinicus minor dexter), nach Marchischer Methode. Leitz Obj. 7. Ocul. I. T. L. $160 \mathrm{~mm}$.

Fig. 8. Katze No. 5. Längsschnitt durch den linken grossen Eingeweidenerven (Splanchinicus major sinistera), nach Marchischer Methode. Leitz Obj. 7. Ocul. I. T. L. $160 \mathrm{~mm}$. 


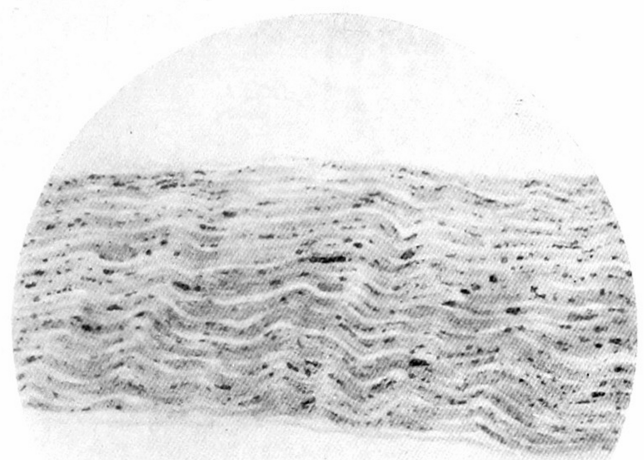

Fig. 1.

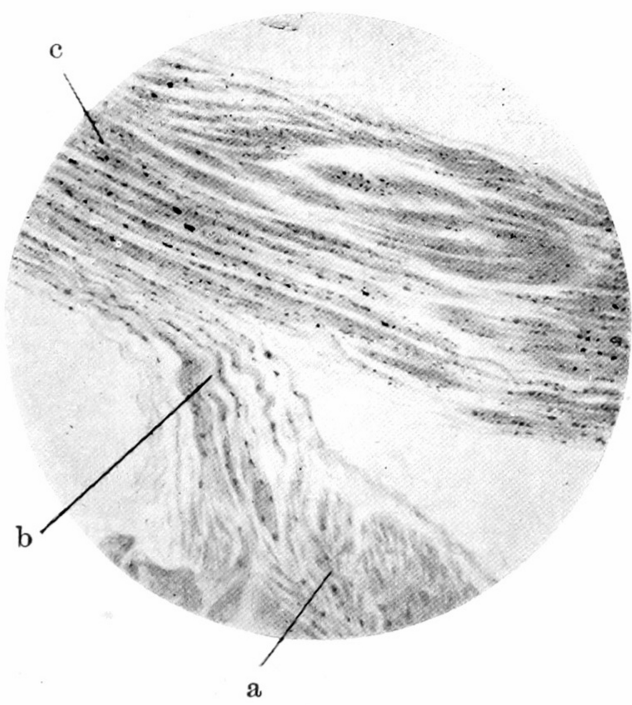

Fig. 3.

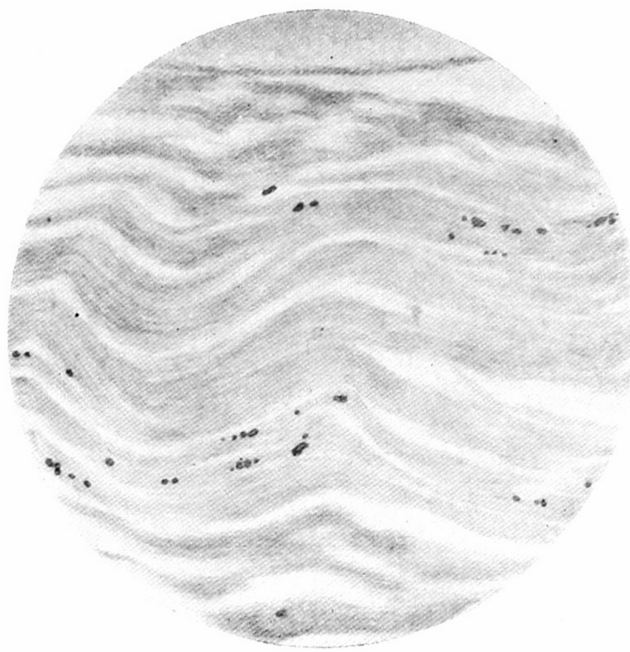

Fig. 2.

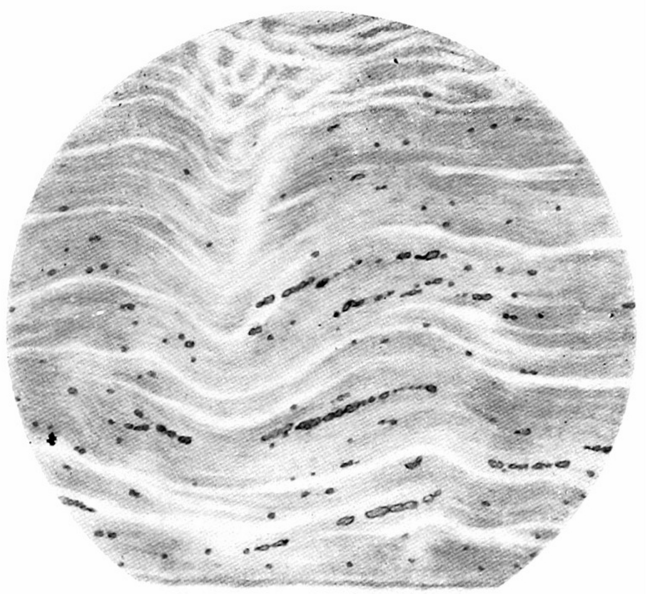

Fig. 4.

Y. Iwama. 


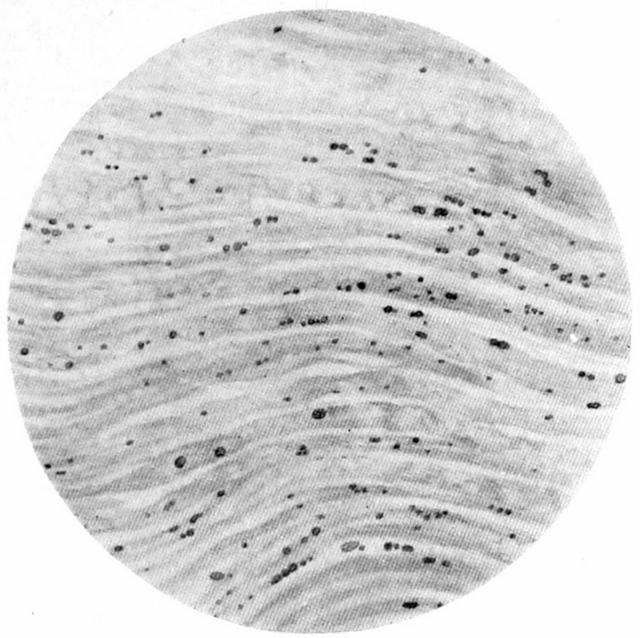

Fig. 5.

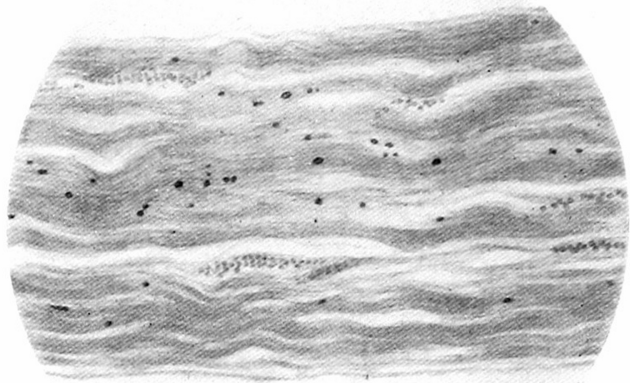

Fig. 6.

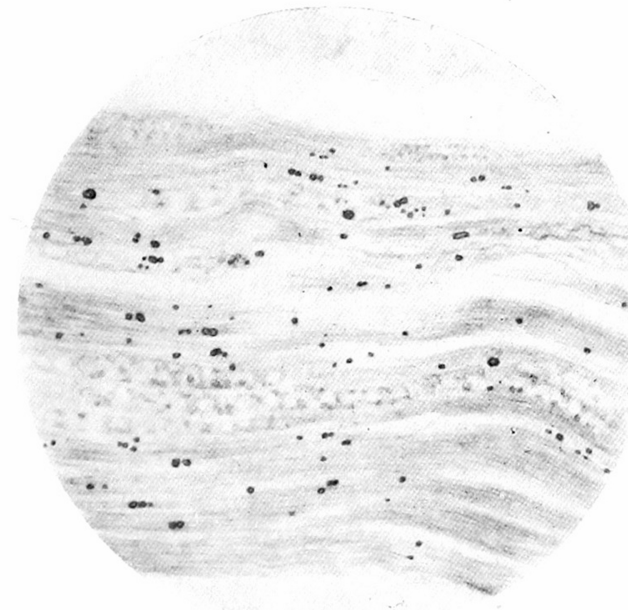

Fig. 7.

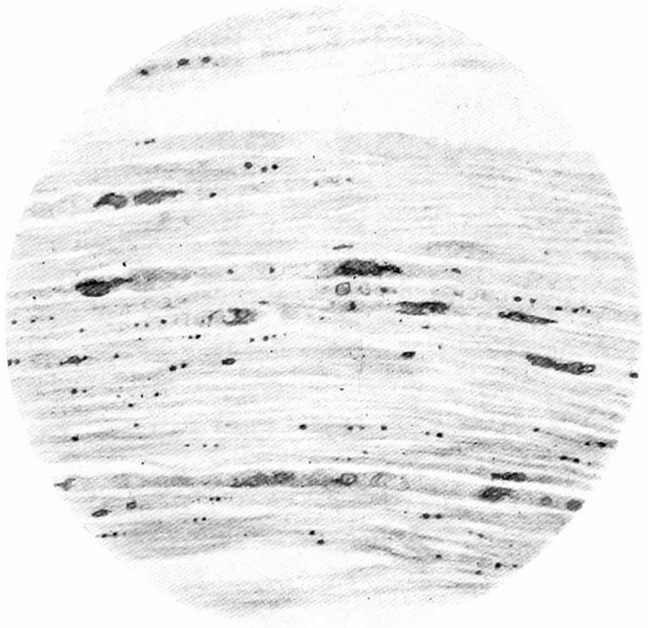

Fig. S.

Y. Iwama. 